Patients with admission $6 \mathrm{MWD}=100 \mathrm{~m}$ had mean improvement $62.2 \mathrm{~m}$ ( \pm 73.1$)$. MBI improved from $69.1( \pm 13.9)$ to $82.1( \pm 12.7)(\mathrm{p}=0.001)$. Patients with MBI 75 on admission had mean improvement of $16.2( \pm 11.7) \quad(p=0.05) .30$ day readmissions for non-infective exacerbations was $15.6 \%$ (vs $29.2 \%$ from historical data). Median duration to exacerbation, death, or censure was 116.5 (IQR 53-206) days. Mean number of issues identified by COAT was $5.6( \pm 2.5), 80.3 \%$ were improved or resolved before discharge.

Conclusion ICARE is a novel inpatient dyspnea support service that improves functional capacity and exercise tolerance, identifies and treats co-morbid medical conditions, and potentially reduces 30 day re-admission to tertiary institutions.

\section{THINKING AHEAD ABOUT MEDICAL TREATMENTS IN ADVANCED ILLNESS: THE COMPLEXITIES OF SUPPORTING DIVERSE COMMUNITIES IN LEICESTERSHIRE}

Zoebia Islam, Lucy Taylor, Helen Eborall, Christina Faull. LOROS, The Leicestershire and Rutland Hospice; University of Leicester

\subsection{6/bmjspcare-2018-ASPabstracts.72}

Background Advance care planning (ACP) supports people who are seriously ill to be cared for in the way, and in the place that they prefer. Yet, evidence suggests there are lower levels of ACP in people from Black, Asian and Minority Ethnic (BAME) communities. Little is known about how the model of resuscitation decision-making fits with the social, cultural and religious values and beliefs of BAME groups. Health care professionals (HCPs) also report a lack of confidence in having culturally appropriate discussions with BAME patients and their families. Equipping professionals to be more confident about such ACP discussions may lead to achieving patient preferences.

Aims This study explores professional views and experiences of ACP with patients from BAME backgrounds. With a focus on making decisions about resuscitation, it aims to identify barriers and enablers and person-centred outcomes to such discussions and provide evidence for training professionals.

Methods Thematic analysis of qualitative semi-structured interviews with HCPs across primary, secondary and tertiary care in Leicester, including GPs, hospital doctors and nurses.

Results There was an emphasis on building rapport, the timing of discussions and navigating communication barriers. Barriers to decision-making included: patients' ${ }^{6}$ and their families understanding of both prognosis and resuscitation; and differing values amongst generations of migrants. Professionals struggled with how to find a balance between acting in a non-discriminatory way whilst respecting cultural differences. Patients and family members, who wished to prolong life at all costs, commonly framed religiously as the sanctity of life, was described as a key challenge to discussions. Most HCPs highlight the need for further training and/or support.

Conclusion There are significant barriers for HCPs when discussing resuscitation decisions with people from BAME communities. This increases the complexity of navigating ACP and achieving patients' preferences. HCPs would benefit from further training and support.

\section{USING A QUALITY IMPROVEMENT APPROACH TO IMPROVE TREATMENT ESCALATION PLANS AND REDUCE CARDIAC ARRESTS AT A LARGE ACUTE NHS TRUST}

Adam Hurlow, Craig Pattison, Alison Cracknell, Anna Winfield, Sherena Nair. Leeds Teaching Hospitals NHS Trust

\subsection{6/bmjspcare-2018-ASPabstracts.73}

Background Cardiopulmonary resuscitation (CPR) is effective for a minority of patients, with survival to discharge of less than 20\%. A recent UK review of in-hospital CPR attempts identified failure to recognise patients at risk of cardiac arrest, discuss treatment escalation plans (TEPs) including CPR, and make do not attempt CPR decisions.

Methods In 2014, Leeds Teaching Hospitals NHS Trust (LTHT) established a quality improvement (QI) collaborative to improve the care of patients at risk of clinical deterioration and reduce avoidable deterioration or inappropriate CPR. It consisted of 14 pilot wards across specialty areas, supported by a multi-disciplinary faculty including Palliative Care.

Three key drivers for change were identified, including a work-stream focussed on timely TEPs for patients nearing the end of life. Over 12 months, pilot wards developed and tested improvement ideas. In June 2015, a bundle of five key interventions, including a TEP sticker and decision prompts, safety huddles and post-CPR debrief, was tested successfully across the 14 wards. A staggered trust-wide roll out of the bundle started in March 2016.

Results Statistical process control charts have shown a sustained and significant $25 \%$ reduction in cardiac arrest calls across LTHT, and a 32\% reduction at the Saint James's University Hospital Site. This equates to 87 fewer cardiac arrests annually across the Trust than in 2015 .

On pilot wards the proportion of patients with a treatment escalation plan and a CPR decision increased by $125 \%$ and $72 \%$, respectively. The Trust incidence of cardiac arrests per 1000 admissions at SJUH is now 25\% lower than the national average.

Conclusion A QI collaborative approach, empowering ward level innovation, with expert faculty support, can improve recognition of patients at risk of cardiac arrest, change behaviours and increase the number of patients with TEPs including CPR decisions; leading to a statistically significant reduction in cardiac arrests.

\section{DEVELOPING A STUDY INTERVENTION: A REALIST REVIEW AND CONSENSUS WORKSHOPS TO DEVELOP THE NAMASTE CARE INTERVENTION FOR PEOPLE WITH ADVANCED DEMENTIA PRIOR TO A FEASIBILITY STUDY USING A CLUSTER RANDOMISED CONTROLLED TRIAL IN NURSING CARE HOMES}

Catherine Walshe, Julie Kinley, Claire Goodman, Frances Bunn, Jennifer Lynch, Rachel Sharpe, Nancy Preston, Shakil Patel, Katherine Froggatt. Lancaster University, University of Hertfordshire, St. Christopher's Hospice

\subsection{6/bmjspcare-2018-ASPabstracts.74}

Background Clear intervention specification is important, but often absent or incomplete in study reports. Namaste Care is 